\title{
Evaluation of the LTE Positioning Capabilities Under Typical Multipath Channels
}

\author{
José A. del Peral-Rosado, José A. López-Salcedo \\ and Gonzalo Seco-Granados \\ Universitat Autònoma de Barcelona (UAB) \\ Bellaterra, Spain \\ Email: \{JoseAntonio.DelPeral, Jose.Salcedo, \\ Gonzalo.Seco\}@uab.cat
}

\author{
Francesca Zanier \\ and Massimo Crisci \\ European Space Agency (ESA) \\ Noordwijk, The Netherlands \\ Email: \{Francesca.Zanier, Massimo.Crisci\}@esa.int
}

\begin{abstract}
The Long Term Evolution (LTE) is a mobile communication standard that is receiving significant attention, and especially offers positioning capabilities by specifying a dedicated downlink signal, i.e. the positioning reference signal (PRS). Thus, this technology can improve the location of mobile terminals operating in harsh environments, such as urban or indoor scenarios. This paper presents a study of the impact of the channel on the positioning capabilities of LTE with respect to the signal bandwidth. For that purpose, typical channel models, such as those recommended by the International Telecommunication Union (ITU), are used to obtain timing error distributions by means of the histogram of maximum likelihood estimates. The results obtained represent the worst-case scenario since the applied estimation process does not consider the presence of the multipath channel. The dependency of the timing error distributions with respect to the type of channel model is also analysed.
\end{abstract}

\section{INTRODUCTION}

Most localization applications are nowadays based on Global Navigation Satellite Systems (GNSS). However, the robustness of mass-market GNSS receivers is compromised in challenging environments, such as indoor or urban scenarios. In these circumstances, the presence of blocking obstacles and propagation disturbances prevent them from observing the expected perfect clear-sky conditions that were assumed in the nominal design of the system. Complementary systems are usually proposed to assist the operation of GNSS systems.

Traditionally, cellular networks have provided the necessary assistance data to improve the overall performance, i.e. assisted-GNSS (A-GNSS), or estimated roughly the user position based on the cell radius, i.e. Cell-ID method. But recently, new positioning capabilities have been incorporated in order to satisfy two main drivers: legal mandates for location identification of emergency calls (e.g. E911 in US or E112 in Europe), and commercial applications or location-based services (LBS), such as navigation, advertising or social media. In this sense, the Long Term Evolution (LTE) technology, specified by the $3^{\text {rd }}$ Generation Partnership Project (3GPP) consortium [1], has defined a dedicated downlink signal for Observed Time Difference of Arrival (OTDoA) positioning, i.e. the positioning reference signal (PRS). In fact, the LTE downlink is defined by a multicarrier Orthogonal Frequency Division Multiplexing (OFDM) signal, which is well-known signal in wireless communications because of its flexibility, spectral efficiency and robustness against frequency-selective fading introduced by multipath, among other advantages with respect to traditional single-carrier signals.

Multipath is certainly a major source of positioning error, especially in indoor and urban areas, where non-line-of-sight (NLOS) conditions are predominant. This topic has received special attention for years within the GNSS community, due to the degradation that NLOS introduces in terms of time delay estimation accuracy. In contrast, terrestrial positioning systems have not addressed significantly this topic since the terrestrial wireless technologies have traditionally focused on communication purposes, and only recently has started to analyse this issue in the literature. Focusing on the time delay estimation (TDE), ultra-wideband (UWB) is an example of system that has been used for localization purposes in dense multipath environments, as shown in [2]. Focusing on other terrestrial systems, the Digital Video Broadcasting (DVB) systems, which is based on OFDM modulation, have been recently studied for signals-of-opportunity (SoO) applications, such as in [3] and [4]. These studies provide positioning results obtained with channel measurements that can lead to realistic assessments of the channel impact. In the case of LTE, studies using measurements campaigns can also be found, such as in [5] with the positioning reference signal or in [6] with a GNSS hybridisation. The resulting multipath impact on the positioning error is usually assessed by using simple metrics, such as the mean delay or center of gravity of the power delay profile, as it is described in [7] and [8]. However, these metrics may not characterize precisely the accuracy impact for every possible bandwidth, and they should be considered as an approximation, as it is shown in this paper.

Although channel model advances are motivated by International Telecommunication Union (ITU) or the European Cooperation in Science and Technology (COST), few studies, such as in [9], are trying to find the detailed impact of the multipath channel on the positioning capabilities of a terrestrial multicarrier system. Thus, the aim of this paper is to characterize the impact of typical channels on the pseudorange estimation using LTE signals, such as the positioning reference signal (PRS), and the dependency of ranging errors 
produced with respect to the type of channel model. In Section II, a description of the LTE standard is presented by following the preliminary analysis on the LTE signal structure shown in [10]. The typical channel models are described in Section III. The maximum likelihood estimation is introduced in Section IV, which is used to produce the multipath error envelope with a two-ray multipath model in Section V. In Section VI, the timing error distribution of typical channel models is obtained by means of the histogram of the maximum likelihood estimates. The evaluation of these distributions is done for fixed and non-fixed tap delay channels. Finally, we draw the conclusions in Section VII.

\section{LONG TERM Evolution (LTE)}

Long Term Evolution (LTE) moves towards the fourth generation (4G) of mobile communications. Most of its standard, which is driven by 3GPP, has been inherited from the Universal Mobile Telecommunication System (UMTS) in order to maintain backward compatibility. The main new features introduced are the downlink Orthogonal Frequency-Division Multiple Access (OFDMA) and the Multiple Input Multiple Output (MIMO) data transmission. The signal bandwidth is scalable from $1.4 \mathrm{MHz}$ to $20 \mathrm{MHz}$ with a symbol period $T_{s}$ of $66.67 \mu \mathrm{s}$, which corresponds to a subcarrier spacing $F_{s c}$ of $15 \mathrm{kHz}$.

According to the LTE specification [11], the downlink positioning procedure, defined by the OTDoA method, uses the difference in the arrival times of downlink radio signals from multiple base stations (i.e. eNodeBs) to compute the user position. The method relies on a network-based strategy because the eNodeB locations are not provided to the user. First, the user equipment (UE) request assistance information to proceed with the timing measurements. Then, the LTE Positioning Protocol (LLP) transfers the UE measurements to the location server, E-SMLC (Enhanced Serving Mobile Location Center). Based on the UE measurements, the E-SMLC estimates the UE position using a trilateration technique, and this position information is finally sent back to the user.

Regarding to the physical layer, the LTE standard [12] specifies a set of downlink signals based on an OFDM modulation with different time-frequency distributions, whose basic structure is shown in Fig. 1 and 2. Downlink synchronization and reference signals are completely known (like the pilot signals in GNSS), and thus they are suitable for ranging purposes. Especially, the primary and secondary synchronization signal (i.e. PSS and SSS), as well as the cell-specific reference signal (CRS), can be used for signals of opportunity (SoO) applications because they do not require any assistance data. However, LTE follows the typical frequency reuse factor of a cellular network, which is equal to one. Thus, the received serving cell signal interferes with the received neighbour cell signals producing inter-cell interference, and resulting in the near-far effect. In order to obtain proper ranging measurements of the neighbour cells, the LTE standard in Release 9 specifies a positioning reference signal (PRS) that is especially dedicated for positioning purposes and mitigates

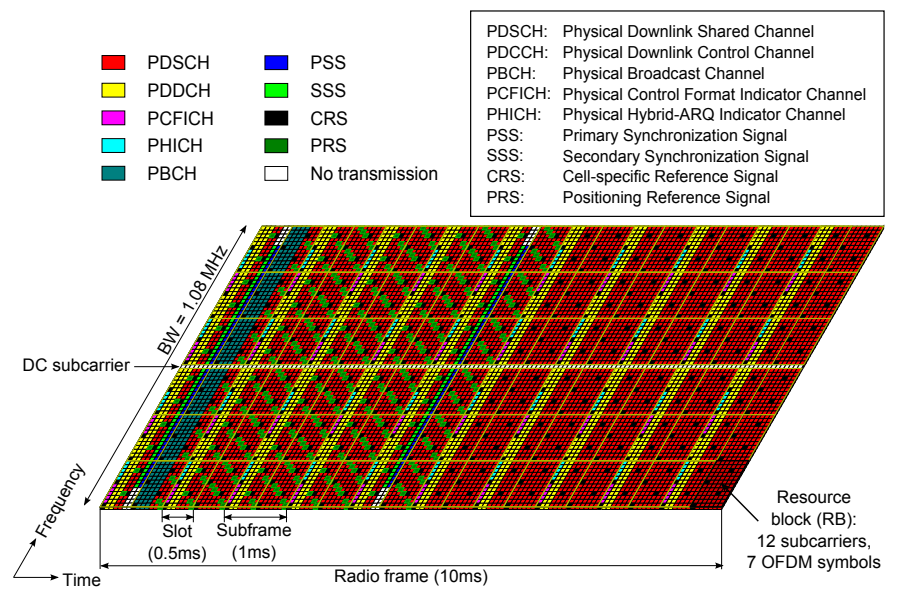

Fig. 1. Time-frequency grid of the LTE signals for $1.4 \mathrm{MHz}$ bandwidth, FDD structure and normal cyclic prefix $(\mathrm{CP})$.

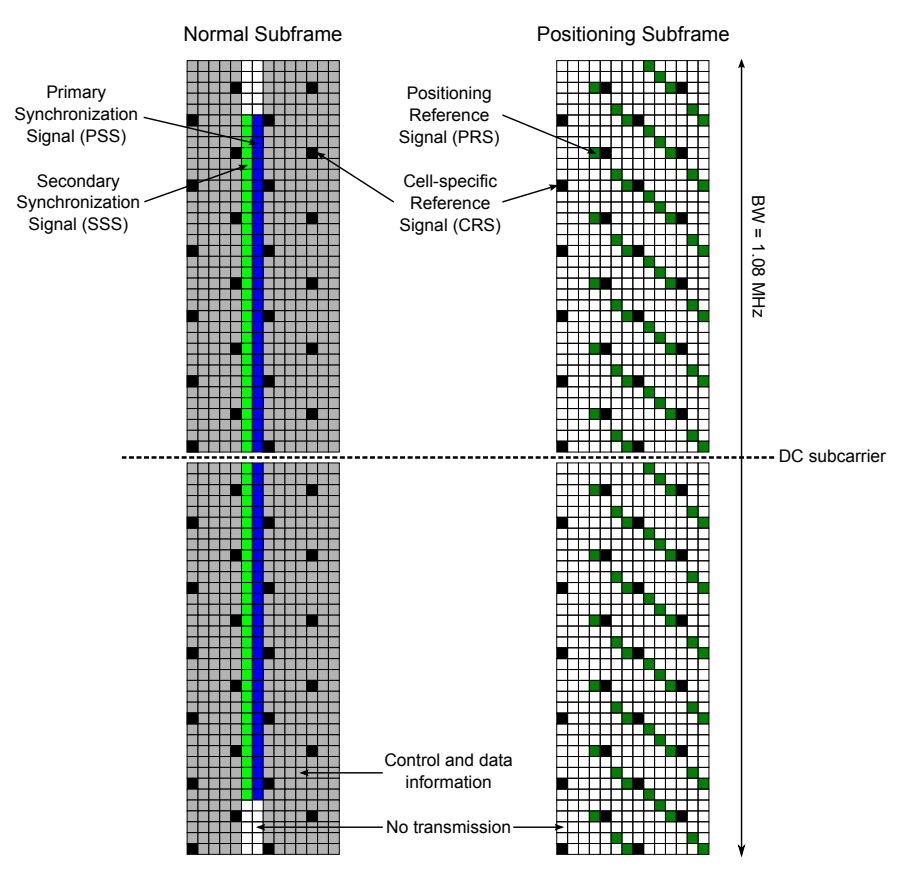

Fig. 2. Example of normal and positioning subframe with 6 resource blocks (RB) for one base station, FDD structure and normal cyclic prefix (CP).

the near-far effect, due to a higher frequency reuse factor (i.e. of six), by shifting one subcarrier position the frequency pilot allocation transmitted by each base station. The main parameters for PRS configuration are shown in Table I. The PRS signal is scattered in time and frequency in the so-called positioning occasion, which allocates consecutive positioning subframes with a certain periodicity. The sophistication of this signal is even higher when the network mutes the PRS transmissions of certain base stations (i.e. PRS muting), in order to further reduce the inter-cell interference. Different interference scenarios are identified in [13], clarifying the achievable accuracy of the PRS signal. 
TABLE I

MAIN PARAMETERS OF THE PRS SIGNAL.

\begin{tabular}{ll}
\hline PRS bandwidth & $1.4,3,5,10,15$ and $20 \mathrm{MHz}$ \\
PRS periodicity & $160,320,640$ or $1280 \mathrm{~ms}$ \\
Consecutive subframes $^{1}, 2,4$, or 6 \\
PRS muting information ${ }^{1}$ & 2, 4, 8, 16 bits \\
PRS pattern & 6-reuse in frequency \\
PRS sequence & Length-31 Gold sequence \\
\hline
\end{tabular}

${ }^{1}$ Number of positioning occasion configured for PRS muting (i.e. bit equal to 0 when PRS is muted).

\section{TyPICAL CHANNEL MODELS}

Propagation channel models are essential tools for simulation and testing of wireless transmission systems. The literature is extensive on this topic, and many standards have recommended channel models for specific propagation environments. These models may characterize path-loss attenuation, shadow fading, and multipath fading. Our interest is focused on the multipath fading propagation conditions present in typical LTE channels, and their impact on the time delay estimation of the signals described in the previous section.

\section{A. Tapped-Delay Line (TDL) Channel Models}

The complexity of the channel model is a major concern when realistic modelling implies a high computational burden. In this sense, the LTE standard adopts simple models to potentially reduce the number of realisations required by the simulations. These models are based on the ITU-R M.1225 [14] recommendation and the 3GPP TS 05.05 [15] specification for GSM, widely used in the context of third generation mobile systems. The ITU and 3GPP models are defined by tapped-delay line (TDL) models, where each tap corresponds to a multipath signal characterized by a fixed delay, relative average power and Doppler spectrum. Their channel impulse response (CIR) can be expressed as

$$
h(\tau ; t)=\sum_{k=1}^{K} a_{k} \delta\left(\tau-\tau_{k}\right),
$$

where $K$ is the number of taps, $\tau_{k}$ is the tap delay relative to the first tap, and $a_{k}$ is the Rayleigh-distributed complex amplitude of the tap, which follows a classical Jakes Doppler spectrum $S(f)$,

$$
S(f) \propto \sqrt{\frac{1}{1-\left(f / f_{D}\right)^{2}}}, \quad \text { for } f \in\left[-f_{D}, f_{D}\right],
$$

being $f_{D}$ the maximum Doppler shift.

Particularly, the 3GPP consortium agreed, in [16], on the use of the Pedestrian A and Vehicular A channels from [14], and the Typical Urban (TU) channel from [15], in order to model three basic environments characterized by a low, medium and large delay spread, respectively. Nevertheless, they were designed for a $5 \mathrm{MHz}$ operating bandwidth, and an apparent periodicity appears in their frequency correlation properties for higher bandwidths [17]. Thus, the LTE standard adopts
TABLE II

LTE TAPPED-DELAY LINE CHANNEL MODELS PARAMETERS.

\begin{tabular}{ccccccc}
\hline \multirow{2}{*}{$\begin{array}{c}\text { Tap } \\
\text { no. }\end{array}$} & \multicolumn{2}{c}{ EPA channel } & \multicolumn{2}{c}{ EVA channel } & \multicolumn{2}{c}{ ETU channel } \\
& $\tau(\mathrm{ns})$ & SMR $(\mathrm{dB})$ & $\tau(\mathrm{ns})$ & SMR $(\mathrm{dB})$ & $\tau(\mathrm{ns})$ & SMR $(\mathrm{dB})$ \\
\hline 1 & 0 & 0.0 & 0 & 0.0 & 0 & -1.0 \\
2 & 30 & -1.0 & 30 & -1.5 & 50 & -1.0 \\
3 & 70 & -2.0 & 150 & -1.4 & 120 & -1.0 \\
4 & 90 & -3.0 & 310 & -3.6 & 200 & 0.0 \\
5 & 110 & -8.0 & 370 & -0.6 & 230 & 0.0 \\
6 & 190 & -17.2 & 710 & -9.1 & 500 & 0.0 \\
7 & 410 & -20.8 & 1090 & -7.0 & 1600 & -3.0 \\
8 & & & 1730 & -12.0 & 2300 & -5.0 \\
9 & & & 2510 & -16.9 & 5000 & -7.0 \\
\hline
\end{tabular}

an extension of the ITU and 3GPP models by following the procedure described in [17], resulting in the Extended Pedestrian A (EPA), Extended Vehicular A (EVA) and Extended Typical Urban (ETU) channel models. The main parameters of these models are specified in TS 36.101 [18] and TS 36.104 [19], and shown in Table II. These specifications also define maximum Doppler shifts for each model to represent low, medium and high mobile conditions. Finally, the TDL models can be applied to multiple antenna schemes by introducing spatial correlation matrices, as it is discussed in [20], resulting on a simple LTE MIMO channel model.

\section{B. Geometric-based Stochastic Channel Models (GSCM)}

The LTE channel can also be modelled with geometricbased stochastic channel models (GSCM). These are more complex models based on the geometry between base station, mobile station and scatterers, following a stochastic construction. The GSCM models are widely adopted for MIMO channel modelling, e.g. COST 259 channel model [21], COST 273 channel model [22], COST 2100 channel model [23], 3GPP spatial channel model (SCM) [24], or the WINNER channel model [25]. Indeed, the ITU-R M.21351 [26] recommendation for the evaluation of IMT-Advanced systems is based on the WINNER channel model, which is able to operate on bandwidths from $5 \mathrm{MHz}$ to $100 \mathrm{MHz}$. According to this recommendation, the deployment scenarios are classified as indoor hotspot, urban micro-cell, urban macrocell and rural macro-cell. Depending on the scenario selected, large-scale parameters, such as delay spread, angle spread or shadow fading, are randomly generated following the distributions specified in Table A1-7 of [26]. Then, the small-scale parameters, such as delay, power, Angle-of-Arrival (AoA) and Angle-of-Departure (AoD), are randomly distributed for each cluster of propagation rays (i.e. rays with similar delay and directions). Both large- and small-scale parameters are fixed during each channel segment (i.e. the so-called drop). Finally, the time-variant channel realisations of a drop are generated according to the random initial phases of the scatterers. A scheme of the GSCM channel model is shown in Fig. 3. 


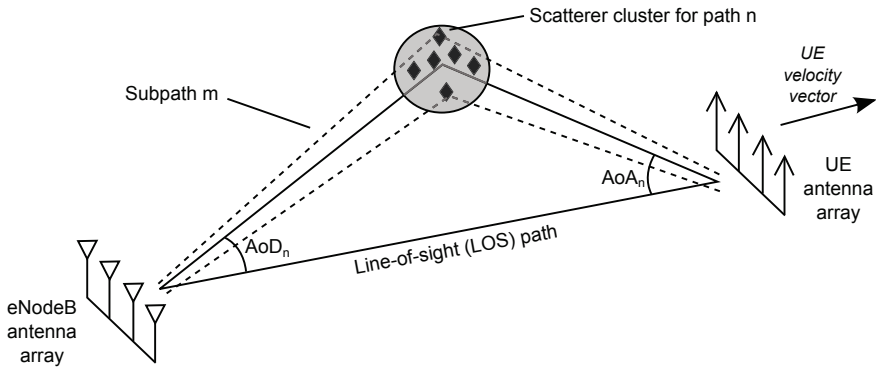

Fig. 3. Scheme of the geometry-based stochastic channel model.

\section{Maximum Likelihood Estimation (MLE)}

Let us define the OFDM baseband signal format for one symbol used in the LTE downlink (without CP) as

$$
x[n]=\sqrt{\frac{2 \cdot C}{N_{c}}} \sum_{k \in \mathcal{N}_{a}} p_{k} \cdot d_{k} \cdot \exp \left(j \frac{2 \pi n k}{N_{c}}\right),
$$

where $C$ is the power of the band-pass signal, $N_{c}$ is the number of subcarriers (excluding unused DC subcarrier), $\mathcal{N}_{a}$ is the subset of active pilot subcarriers $N_{a}$, which must satisfy $N_{a} \leq N_{c}, d_{k}$ are the symbols, and $p_{k}^{2}$ is the relative power weight of subcarrier $k$, which is constrained by $\sum_{k} p_{k}^{2}=N_{c}$ to give the nominal signal power $C$. In the presence of additive white Gaussian noise (AWGN), the received signal $r[n]$ can be defined as

$$
r[n]=x\left[n ; n_{\tau}\right]+w[n],
$$

where the discrete time delay (in samples) is $n_{\tau}=\tau \cdot F_{s}$, being $F_{s}$ the sampling frequency, and $w[n]$ the noise component. Thus, the maximum likelihood estimation (MLE) is based on the correlation of the received signal $r[n]$ with a shifted and conjugated version of the reference signal $x[n]$, which is assumed periodical (i.e. circular correlation), in order to find the correlation peak. The resulting correlation between the received and the transmitted signal is defined by

$$
R_{r x}(\tau) \doteq \sum_{n=0}^{N_{c}-1} r[n] \cdot x_{c}^{*}\left[n+n_{\tau}\right],
$$

where $x_{c}[n]$ is a circular shifted version of the original $x[n]$, resulting in the matched filter of the OFDM signal, whose estimated delay can be expressed as

$$
\hat{\tau}=\frac{T_{s}}{N_{c}} \arg \max _{\tau}\left\{\left|R_{r x}(\tau)\right|^{2}\right\},
$$

where $\tau$ is the time delay in seconds. In Fig. 4, the autocorrelation function of $x_{c}[n]$ is shown for different bandwidth configurations of the LTE positioning reference signal (PRS) using only one OFDM symbol. As it can be noticed, the bandwidth is denoted according to the number of resource blocks (RB) occupied by the PRS signal in the frequency domain (i.e. $180 \mathrm{kHz}$ per RB). Thus, the maximum likelihood estimate is obtained by measuring the time delay corresponding to the maximum of the correlation function.

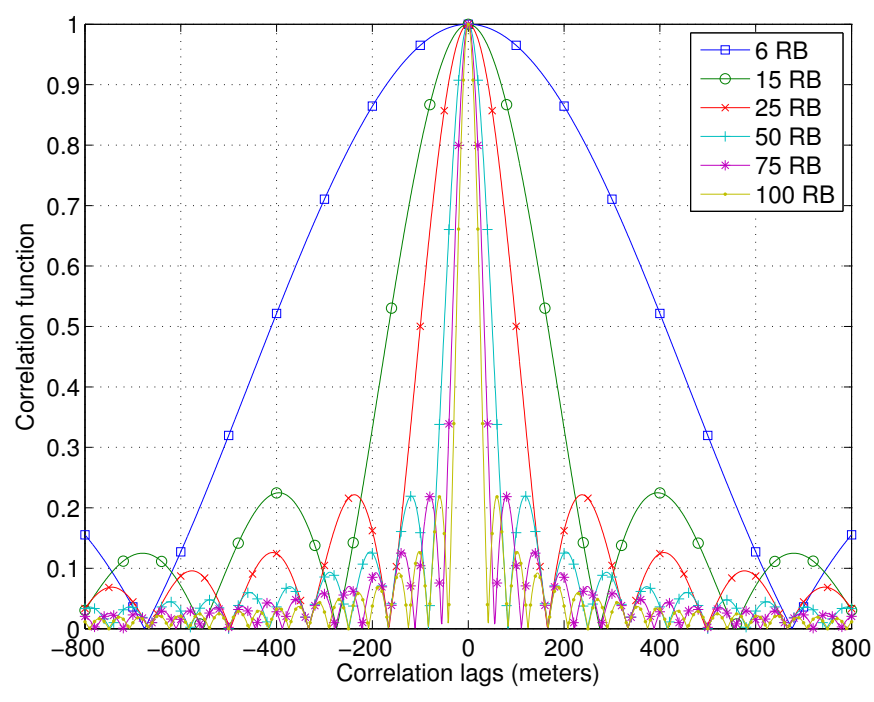

Fig. 4. Autocorrelation function of the LTE PRS signal for the different standard bandwidth and one OFDM symbol.

\section{Multipath ERror EnVElope (MPEE)}

The impact of the multipath channel on the positioning error can be preliminary studied by means of the multipath error envelope (MPEE). This metric is based on the evaluation of the time delay error produced when adding to the line-of-sight (LOS) signal an artificial multipath signal, which is generated with specific delay, power and phase.

Considering (5) and (6), the MPEE is produced by means of the maximum likelihood estimation and using a two-ray multipath model (i.e. LOS and multipath signal), as it is shown in Fig. 5. Firstly, the signal-to-multipath ratio (SMR) is changed for the smallest PRS bandwidth, in Fig. 5(a), to assess the impact of the multipath power. The results are obtained for the destructive and constructive contribution of the multipath, that is, when the multipath ray is in-phase (solid line) and counter-phase (dashed line), respectively. The taps delays of the ETU channel are also depicted (dotted line) to highlight the multipath ray error for those delays. Secondly, the different PRS bandwidth are tested for an SMR equal to $1 \mathrm{~dB}$, in Fig. 5 (b), to confirm the multipath error reduction as the bandwidth increases.

As it can be noticed, the maximum delay error produced by a single multipath ray can be found easily for different scenarios with the multipath error envelope. Nevertheless, the multipath error assessment is more complicated in a realistic channel due to the increase on the number of multipath rays. This observation suggests that another metric should be found in order to measure the impact of a certain multipath channel on the time delay.

\section{TIMING ERROR HISTOGRAM}

An alternative tool on the assessment of the multipath impact is the probability density function (PDF) of the time delay error for a specific channel. The PDF of the timing error can help us to find the maximum error that bounds the accuracy of 


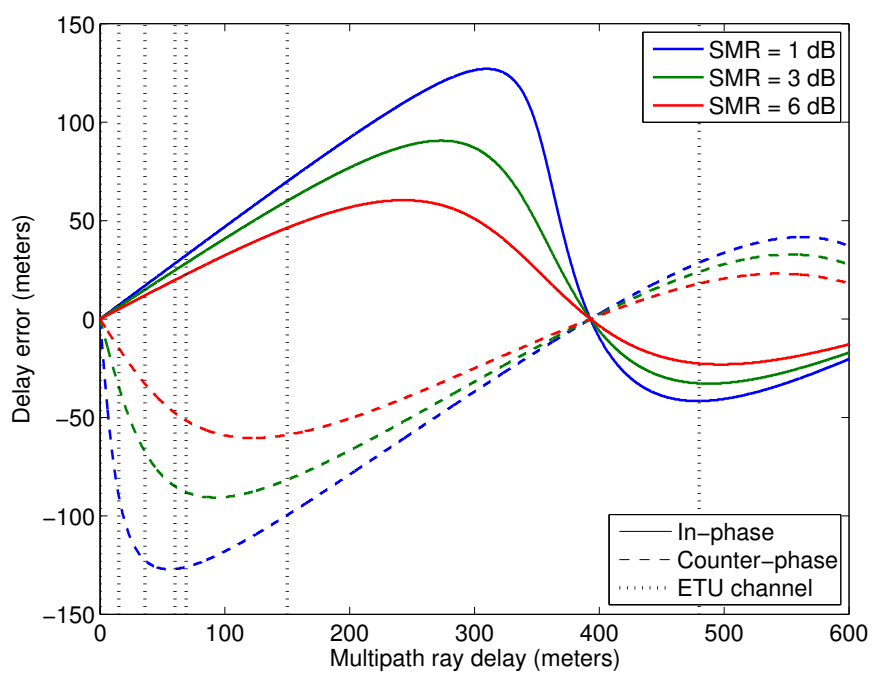

(a) Different SMR for $6 \mathrm{RB}$ bandwidth

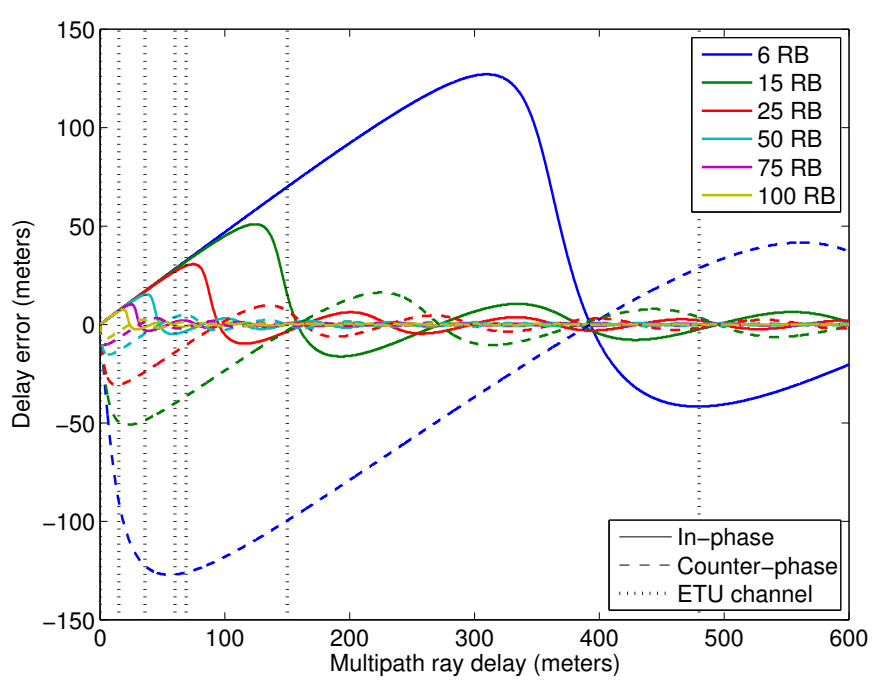

(b) Different PRS bandwidth for SMR equal to $1 \mathrm{~dB}$

Fig. 5. Multipath error envelope (MPEE) for the LTE PRS signal.

the LTE positioning scheme. For that purpose, a large number of channel realisations is necessary to cover the majority of possible timing errors produced by the multipath channel, from the statistical point of view. The timing errors are obtained by computing the MLE of the time delay for each channel realisation. The histogram of the resulting timing errors finally shows the impact of the channel on the positioning.

\section{A. Tapped-Delay Line (TDL) Channel Models}

The first case of interest is based on the evaluation of the traditional TDL channel models specified in LTE (i.e. EPA, EVA and ETU channel models). Since their multipath delays are constant over time, we can measure the impact produced by the complex amplitude variation of every multipath ray.

The Communications System Toolbox ${ }^{\mathrm{TM}}$ provided in MATLAB is used to perform the simulations. This toolbox contains the stdchan function to simulate multipath fading channels, where the LTE channel parameters of Table II can be introduced. In order to cover the maximum number of multipath ray combinations, a fast fading scenario is selected by defining a maximum Doppler shift of $300 \mathrm{~Hz}$. The resulting histograms are shown in Fig. 6 for 20000 channel realisations and using a Savitzky-Golay FIR smoothing filter. The power delay profile (PDP) of the EPA, EVA and ETU channels is also added in order to identify which ray or group of rays may have caused a certain timing error.

As it could be expected, the PDP distribution characterizes the impact of the channel on the timing error. For instance, the histograms for EPA channel, in Fig. 6(a), are centred on the multipath rays that concentrate more energy. Thus, many authors, such as [7] and [8], have used the mean delay, or the so-called center of gravity, of the PDP to measure the delay error by computing a weigthed average of the taps delays using the taps squared amplitude, defined by

$$
\bar{\tau}=\frac{\sum_{k=1}^{K} \tau_{k}\left|a_{k}\right|^{2}}{\sum_{k=1}^{K}\left|a_{k}\right|^{2}} .
$$

Nevertheless, this metric has to be carefully used because it may not characterize the maximum likelihood timing estimate obtained for the actual multipath channel. The main constraint is posed by the signal bandwidth, which is not considered in the metric but which determines the ultimate shape of the correlation function. As it is shown in Fig. 4, the main lobe of the correlation function is narrowed when increasing the bandwidth, and thus the separation between multipath rays has to be lower in order to jointly contribute to the timing error. This effect can be especially noticed for high bandwidths, where one can easily identify the multipath rays in the timing error distributions, as it shown in Fig. 6.

A similar metric, which may vary due to the bandwidth, can be obtained by computing the mean delay error or center of gravity of the histogram of the MLE $f(i)$, defined as

$$
\bar{\tau}_{\varepsilon}=\frac{\sum_{i=1}^{L} \tau_{\varepsilon}(i) f(i)}{\sum_{i=1}^{L} f(i)},
$$

where $\tau_{\varepsilon}(i)$ is the timing error for the histogram interval $i$, and $L$ is the number of histogram intervals. As it is compared in Table III, the results of this metric $\bar{\tau}_{\varepsilon}$ depend on the signal bandwidth, while the mean delay or center of gravity of the power delay profile does not change. Thus, the results obtained can lead to a more accurate assessment of the positioning capabilities. In addition, the resulting histograms can be used to identify the impact of each multipath ray on the ranging error. Nevertheless, the calculation of the timing error histogram of the MLE implies a high computation burden due to the high number of realizations required. 
TABLE III

MEAN DELAY ERROR OF THE PDP AND OF THE Histograms OF Fig. 6 FOR LTE PRS SIGNALS IN TDL CHANNEL MODELS.

\begin{tabular}{ccccccc}
\hline \multirow{2}{*}{$\begin{array}{c}\text { Bandwidth } \\
(\mathrm{RB})\end{array}$} & \multicolumn{2}{c}{ EPA channel } & \multicolumn{2}{c}{ EVA channel } & \multicolumn{2}{c}{ ETU channel } \\
$\bar{\tau}(\mathrm{m})$ & $\bar{\tau}_{\varepsilon}(\mathrm{m})$ & $\bar{\tau}(\mathrm{m})$ & $\bar{\tau}_{\varepsilon}(\mathrm{m})$ & $\bar{\tau}(\mathrm{m})$ & $\bar{\tau}_{\varepsilon}(\mathrm{m})$ \\
\hline 6 & 13 & 13 & 47 & 55 & 59 & 61 \\
15 & 13 & 13 & 47 & 49 & 59 & 58 \\
25 & 13 & 13 & 47 & 46 & 59 & 55 \\
50 & 13 & 13 & 47 & 44 & 59 & 55 \\
75 & 13 & 13 & 47 & 44 & 59 & 58 \\
100 & 13 & 13 & 47 & 42 & 59 & 60 \\
\hline
\end{tabular}

\section{B. Geometric-based Stochastic Channel Models (GSCM)}

The second case of interest studies tap delay variations of the multipath rays. The tapped-delay line (TDL) models, used in the previous section, only change the complex amplitude of the taps for every channel realisation, maintaining a fixed tap delay distribution. Thus, more complex models, such as GSCM models, are used to compare fixed and non-fixed taps delays, in order to assess the impact of the tap delay position and resolution.

The WINNER channel models [25] are chosen for this evaluation because their higher complexity provides more flexibility on the simulation test. These models increase the number of paths (i.e. up to 20 paths) leading to rich power delay profiles that can help us to assess different situations. Particularly, the B2 propagation or bad urban micro-cell scenario is used for this study. The WINNER B2 scenario is characterized by a Manhattan-like urban area, where transmitter and receivers are located outdoors surrounded by buildings with the eventual presence of far scatterers. Non-LOS (NLOS) conditions are predominant with user velocities up to $70 \mathrm{~km} / \mathrm{h}$.

In order to produce the channel realisations, the MATLAB code provided in [27] is used. The simulation is simplified to a single link between transmitter and receiver with single isotropic antennas. Although the code computes path losses and shawoding coefficients, they are not applied to the channel in order to focus on the analysis of the multipath components. Time evolution of the taps delays is described in the WINNER specification [25, p.33] by defining simulations of multiple and correlated channel segments. Since a channel segment is a group of realisations with fixed parameters, where phases of rays are only varying, smooth transition between segments allow time evolution of propagation parameters. However, current implementation of WINNER model does not account for the time evolution option. Thus, the evaluation of tap delay variations is simplified in our study by applying a Gaussian distribution to the fixed tap delay positions. The application of the Gaussian distribution does not intend to model a realistic variation of the taps delays, but to assess the impact of the delay variation statistically.

A single channel segment of 40000 realisations is computed, and a sampling grid of 0.25 meters is defined in the delay domain. Since the paths delays are fixed during the whole
TABLE IV

STANDARD DEVIATION AND MEAN DELAY ERROR FOR LTE PRS SIGNALS IN WINNER B2 CHANNEL MODEL, FOR HISTOGRAMS OF FIG. 7.

\begin{tabular}{ccccccc}
\hline \multirow{2}{*}{$\begin{array}{c}\text { Bandwidth } \\
(\mathrm{RB})\end{array}$} & \multicolumn{2}{c}{ Fixed } & \multicolumn{2}{c}{ Non-fixed 1 } & \multicolumn{2}{c}{ Non-fixed 2 } \\
$\sigma_{\varepsilon}(\mathrm{m})$ & $\bar{\tau}_{\varepsilon}(\mathrm{m})$ & $\sigma_{\varepsilon}(\mathrm{m})$ & $\bar{\tau}_{\varepsilon}(\mathrm{m})$ & $\sigma_{\varepsilon}(\mathrm{m})$ & $\bar{\tau}_{\varepsilon}(\mathrm{m})$ \\
\hline 6 & 80 & 157 & 80 & 157 & 80 & 158 \\
15 & 71 & 160 & 71 & 159 & 72 & 160 \\
25 & 68 & 166 & 68 & 167 & 69 & 166 \\
50 & 64 & 176 & 64 & 176 & 68 & 173 \\
75 & 63 & 177 & 64 & 177 & 69 & 174 \\
100 & 64 & 177 & 65 & 176 & 70 & 174 \\
\hline
\end{tabular}

simulation, a Gaussian-distributed delay is artificially added to every existing path for each realisation. Thus, tap delay variations $\tau_{k}(j)$ are defined as

$$
\tau_{k}(j) \sim \mathcal{N}\left(\bar{\tau}_{k}, \sigma_{\tau}^{2}\right)
$$

where $j$ is the channel realisation, $\bar{\tau}_{k}$ is the mean tap delay defined by the channel model, and $\sigma_{\tau}$ is the standard deviation of the artificial delays. Two standard deviations, i.e. $\sigma_{\tau}$ is equal to 5 and 20 meters, are defined to assess two example cases with low and high variations, respectively, which do not model any specific mobile scenario.

Following the same procedure of the previous section, timing error histograms for fixed and non-fixed taps delays cases are simulated considering the WINNER B2 channel model and the different LTE PRS bandwidths, as is shown in Fig. 7. The power delay profile of the three cases is also depicted in Fig. 7(a), 7(b) and 7(c), where the Gaussian distribution of the artificial variation is also introduced. From these results, the relationship between timing errors and energy concentration of the multipath rays can be clearly identified, as in the previous section. But, the variation of the taps delays increases the variance of the timing error distribution, being more difficult to assess the impact of the different taps. This effect is stressed for the case with higher variation (i.e. $\sigma_{\tau}=20$ meters), being more noticeable for high bandwidths where the timing error peaks are wider than in the fixed delay case. As it is shown in Table IV, the standard deviation $\sigma_{\varepsilon}$ of the timing error distribution is a function of both standard deviation of the tap delay variation $\sigma_{\tau}$ and the signal bandwidth, that is, it increases when these two parameters increase. Therefore, a higher variability of the taps delays, either introduced by the receiver mobility or the environment change, produces a wider range of timing error values with different probability that may reduce the precision of the time-delay estimation (TDE) technique under test. This analysis shows the importance of an accurate channel model able to reproduce a realistic time-varying test scenario. As a final remark, the results obtained with the current implementation of the maximum likelihood estimator (i.e. the maximum peak of the correlation function) can still be improved in multipath environments. TDE techniques that consider the presence of the multipath channel are expected to provide better positioning results. 

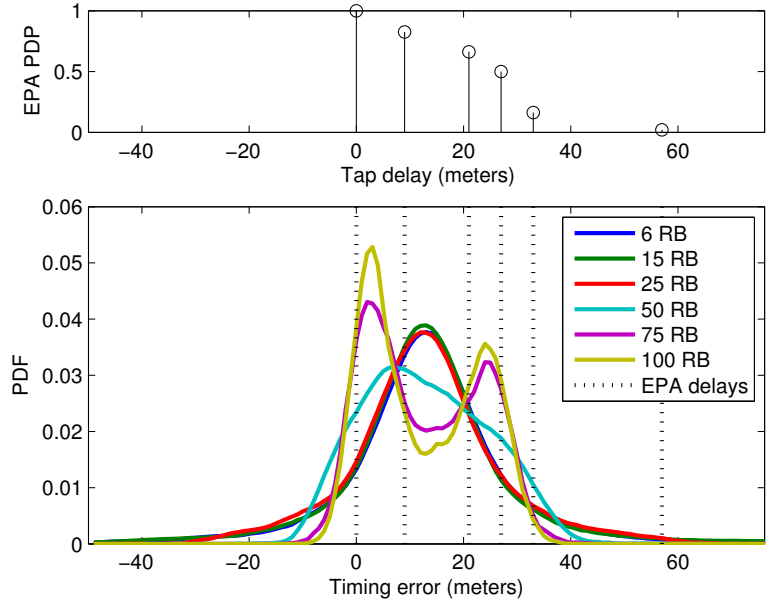

(a) EPA channel model
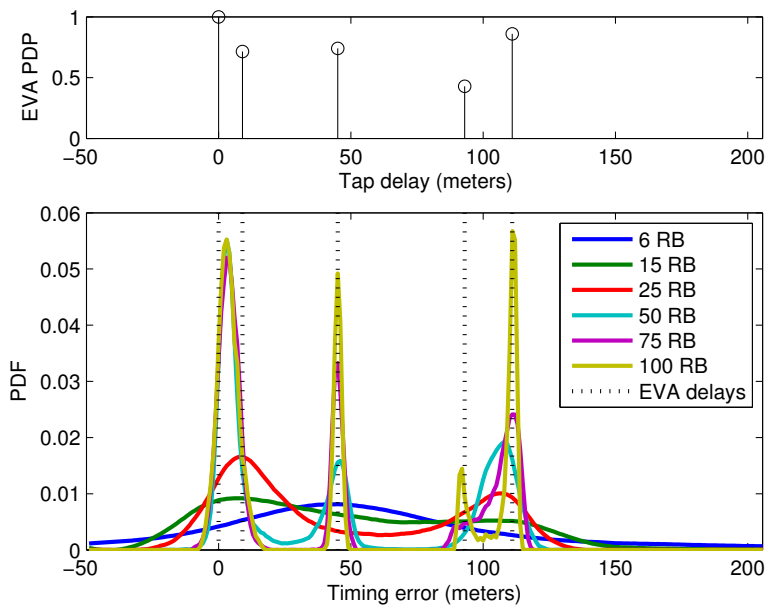

(b) EVA channel model
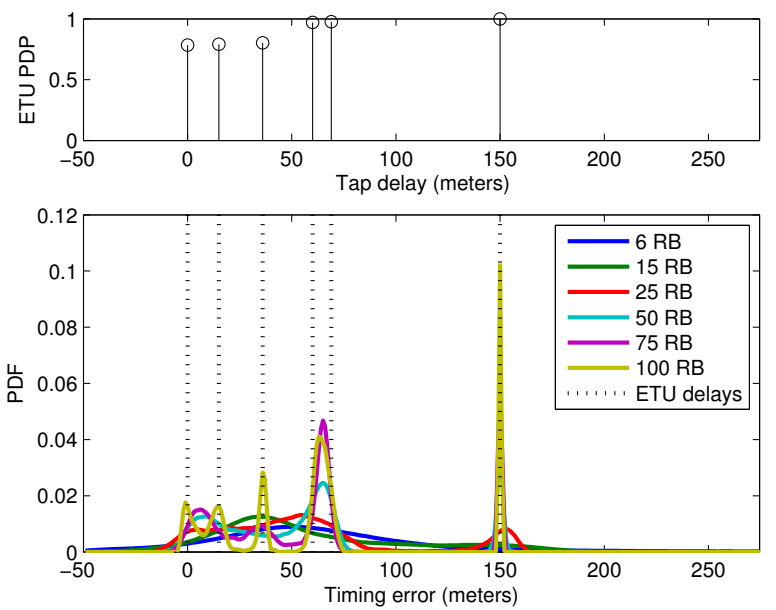

(c) ETU channel model

Fig. 6. Timing error histograms of the MLE for TDL channel models.
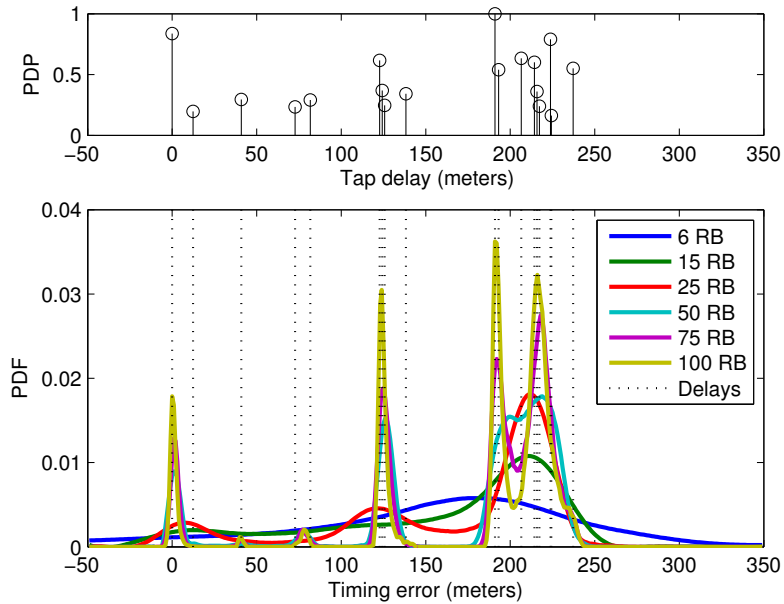

(a) Fixed taps delays
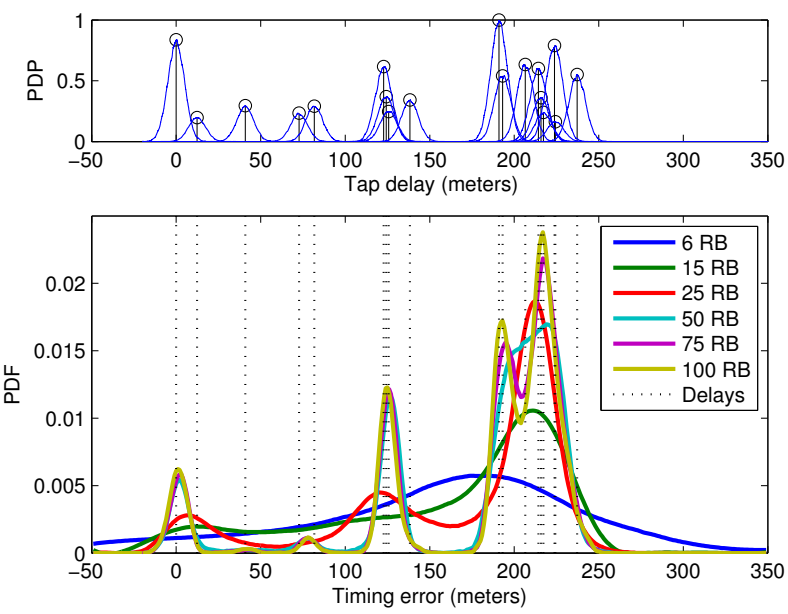

(b) Non-fixed taps delays $\left(\sigma_{\tau}=5 \mathrm{~m}\right)$
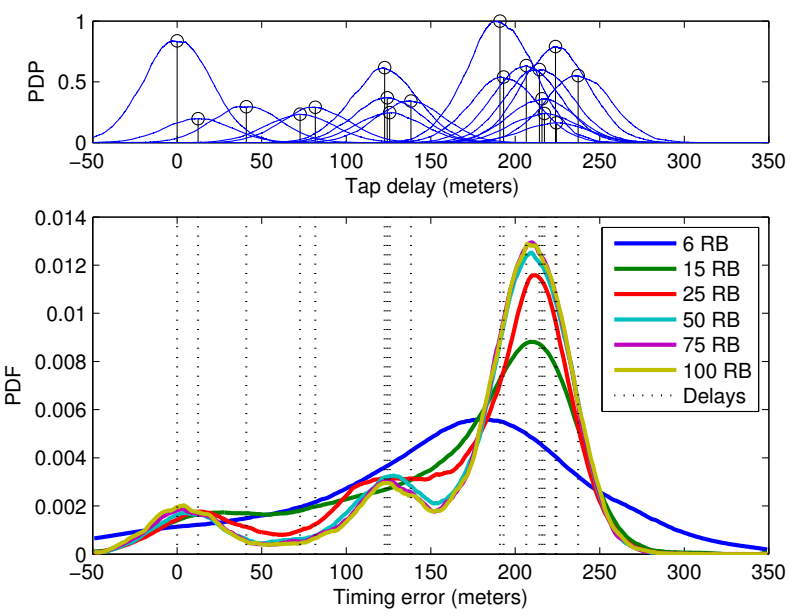

(c) Non-fixed taps delays $\left(\sigma_{\tau}=20 \mathrm{~m}\right)$

Fig. 7. Timing error histograms of the MLE for WINNER B2 channel model. 


\section{CONCLUSION}

The impact of typical channel models, especially of their multipath propagation, on time delay estimation has been assessed for the Long Term Evolution (LTE) positioning capabilities. A two-ray multipath model has been used to preliminary evaluate the influence of the multipath power, multipath delay and signal bandwidth on the positioning error, by means of the multipath error envelope (MPEE). Due to the limitations of this metric, a mean delay error (MDE) has been calculated from the timing error histogram of the maximum likelihood estimation (MLE), which is computed for the traditional tapped-delay line (TDL) channel models specified in LTE and for the WINNER channel model. It is shown that the MDE is function of the LTE signal bandwidth. It implicitly adds information of the signal characteristics, which are not traced by traditional metrics based on the channel model. The state-of-the-art terrestrial channel models are intended for communications purposes, thus the tap delay position is fixed, not having an important effect. However, assuming that the tap delay position might have an impact on the results of the ranging error distributions, the variation of the tap delay have also been analysed by adding as a first approximation a Gaussian distribution on the tap-delay position. Results show that the standard deviation of the timing error distributions is a function of both standard deviation of the Gaussian-distribution of the taps delays and the signal bandwidth. Further evaluation of the multipath impact on the positioning error could be done by realistically modelling the time evolution of the taps delays derived from the user movement in specific scenarios. In addition, the application of techniques that consider the presence of the multipath channel is expected to improve the positioning accuracy obtained.

\section{ACKNOWLEDGMENT}

This work was supported by the European Space Agency (ESA) under the PRESTIGE programme ESA-P-2010-TECETN-01 and by the Spanish Ministry of Science and Innovation project TEC 2011-28219.

\section{REFERENCES}

[1] 3GPP home page. [Online]. Available: http://www.3GPP.org

[2] A. A. D'Amico, U. Mengali, and L. Taponecco, "TOA Estimation with the IEEE 802.15.4a Standard," IEEE Trans. on Wireless Communications, vol. 9, no. 7, pp. 2238-2247, July 2010.

[3] P. Thevenon, S. Damien, O. Julien, C. Macabiau, M. Bousquet, L. Ries, and S. Corazza, "Positioning Using Mobile TV Based on the DVB-SH Standard," in NAVIGATION, vol. 58, no. 2, Summer 2011, pp. 71-90.
[4] D. Serant, O. Julien, L. Ries, P. Thevenon, and M. Dervin, "The Digita TV Case: Positioning Using Signals-of-Opportunity Based on OFDM Modulation," Inside GNSS, Nov./Dec. 2011.

[5] J. Medbo, I. Siomina, A. Kangas, and J. Furuskog, "Propagation channel impact on LTE positioning accuracy: A study based on real measurements of observed time difference of arrival," in Proc. IEEE PIMRC '09, Sep. 2009, pp. 2213-2217.

[6] A. Dammann, E. Staudinger, S. Sand, and C. Gentner, "Joint GNSS and 3GPP-LTE based positioning in outdoor-to-indoor environments performance evaluation and verification," in Proc. ION GNSS, Sep. 2011.

[7] R1-092307, "Analysis of UE Subframe Timing Offset Measurement Sensitivity to OTDoA Performance," 3GPP, Alcatel-Lucent, RAN1$57 \mathrm{bis}$, June 2009.

[8] C. Mensing and A. Dammann, "Positioning with OFDM based communications systems and GNSS in critical scenarios," in Proc. WPNC '08, Mar. 2008, pp. 1-7.

[9] W. Wang, T. Jost, A. Lehner, F. Perez-Diaz, and U.-C. Fiebig, "Towards a Channel Model for Joint GNSS and Mobile Radio Based Positioning," in COST Action IC1004, Oct. 2011.

[10] J. A. Del Peral-Rosado, J. A. López-Salcedo, G. Seco-Granados, F. Zanier, and M. Crisci, "Preliminary analysis of the positioning capabilities of the positioning reference signal of 3GPP LTE," in Proc. 5th Eur. Workshop on GNSS Signals and Signal Processing, Dec. 2011.

[11] 3GPP TS 36.305, Stage 2 functional specification of User Equipment (UE) positioning in E-UTRAN, Std.

[12] 3GPP TS 36.211, Physical Channels and Modulation, Std.

[13] J. A. Del Peral-Rosado, J. A. López-Salcedo, G. Seco-Granados, F. Zanier, and M. Crisci, "Achievable Localization Performance Accuracy of the Positioning Reference Signal of 3GPP LTE," in Proc. International Conf. on Localization and GNSS (ICL-GNSS), June 2012

[14] ITU-R M.1225 International Telecommunication Union, "Guidelines for evaluation of radio transmission technologies for IMT-2000,” 1997.

[15] 3GPP TS 05.05, Radio transmission and reception, Std.

[16] R4-070572, "Proposal for LTE channel models," 3GPP, Ericsson, Nokia, Motorola, Rohde \& Schwarz, RAN4-43, May 2007.

[17] T. Sorensen, P. Mogensen, and F. Frederiksen, "Extension of the ITU channel models for wideband (OFDM) systems," in Proc. IEEE VTC '05, vol. 1, Sept. 2005, pp. 392-396.

[18] 3GPP TS 36.101, User equipment (UE) radio transmission and reception, Std.

[19] 3GPP TS 36.104, Base station (BS) radio transmission and reception, Std.

[20] R4-060334, "LTE Channel Models and simulations," 3GPP, Ericsson, Elektrobit, Nokia, Motorola, Siemens, RAN4-38, Feb. 2006.

[21] A. Molisch, H. Asplund, R. Heddergott, M. Steinbauer, and T. Zwick, "The COST259 Directional Channel Model-Part I: Overview and Methodology," IEEE Trans. on Wireless Communications, vol. 5, no. 12, pp. 3421-3433, Dec. 2006.

[22] L. Correia, Ed., Mobile Broadband Multimedia Networks. Academic Press, 2006.

[23] R. Verdone, Ed., COST 2100 - Pervasive Mobile and Ambient Wireless Communications. Berlin, Germany: Springer, 2011.

[24] 3GPP TS 25.996, Spatial Channel Model for Multiple Input Multiple Output (MIMO) Simulations, Std.

[25] Commission of the European Communities, IST-WINNER II project deliverable D1.1.2, WINNER II Channel Models, Std.

[26] ITU-R M.2135-1 International Telecommunication Union, "Guidelines for evaluation of radio interface technologies for IMT-Advanced," 2008

[27] L. Hentilä, P. Kyösti, M. Käske, M. Narandzic, and M. Alatossava, "MATLAB implementation of the WINNER Phase II Channel Model ver1.1," IST-WINNER II Project, Dec, 2007. 